\title{
Individualización de los métodos de reducción lítica en el Paleolítico antiguo de Bañugues (Gozón, Asturias)
}

\section{Lithic Knapping methods in the Early Palaeolithic site of Bañugues (Gozón, Asturias)}

\author{
David Álvarez Alonso*
}

\begin{abstract}
RESUMEN
El yacimiento de Bañugues es uno de los referentes en el estudio del Paleolítico antiguo en el norte de la Península Ibérica. Excavado a finales de los años 70 del siglo XX y encuadrado en las etapas finales del Achelense, en este artículo se revisa su adscripción cronocultural en función del análisis tecnológico de las cadenas operativas identificadas.
\end{abstract}

\section{PALABRAS CLAVE}

Paleolítico antiguo, Paleolítico inferior, Paleolítico medio, Achelense, Asturias.

\begin{abstract}
Bañugues constitutes a key site for the study of the Early Palaeolithic period in the north area of the Iberian Peninsula since it was excavated towards the end of the 1970 's. This paper focuses on its chronocultural association to the final-Acheulian phase. My revision is based on the technological analysis o identified operational sequences.
\end{abstract}

\section{KEY WORDS}

Early Palaeolithic, Lower Palaeolithic, Middle Paleolithic, Acheulean, Asturias.

* Departamento de Prehistoria y Arqueología, UNED, dalvarez@gijon.uned.es 


\section{INTRODUCCIÓN}

El yacimiento de Bañugues, el cual contiene una secuencia arqueológica que comprende niveles del Achelense y del Asturiense (Rodríguez Asensio, 1980, 1983), se encuentra en el centro del litoral asturiano, al Este del Cabo de Peñas, en una ensenada formada por un valle abierto al mar, recibiendo su nombre del

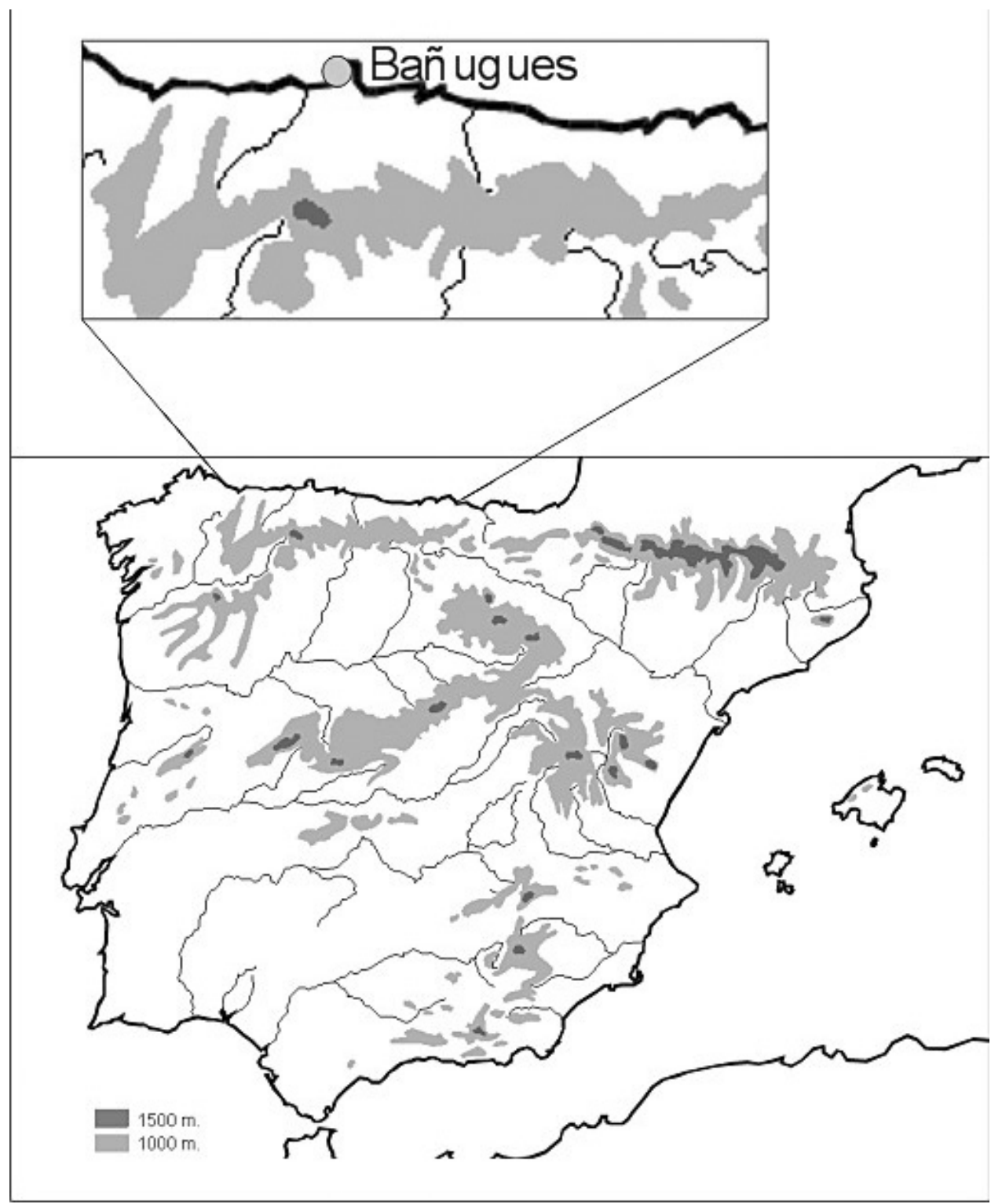

Fig. 1. Localización de Bañugues. 
pueblo en cuyo término se encuentra. Se trata del primer yacimiento con cronología atribuida al Paleolítico inferior excavado en Asturias, y uno de los pioneros en la región Cantábrica, por lo que desde hace décadas es una de las referencias más citadas e ineludibles cuando se aborda la temática del Paleolítico antiguo asturiano, en particular, y cantábrico en general (Rodríguez Asensio, 2004).

Bañugues fue descubierto por Domingo Caramés y Joaquín Manzanares en 1961 (Pérez Pérez, 1975) instante a partir del cual empezó a ser fruto de intensas y constantes recogidas superficiales de material en sus inmediaciones, siendo estos hallazgos atribuidos sucesivamente al Abbevillense (González Fernández, 1968), Achelense y Asturiense (Jordá, 1975), del Abbevillense al Musteriense de tradición achelense (Pérez Pérez, 1975) y del Achelense superior evolucionado al Musteriense de tradición achelense (Rodríguez Asensio, 1978b). Importante también es la referencia que hace Jordá a la edad rissiense de las arcillas de la base del depósito (Jordá, 1975), ya que es un elemento que sin duda reafirma la asignación al Achelense final (Rodríguez Asensio, 1980, 1983).

\section{LAS EXCAVACIONES}

A finales de la década de los 70 del pasado siglo, J. Adolfo Rodríguez Asensio lleva a cabo una serie de campañas arqueológicas (Rodríguez Asensio, 1978a, $1978 b, 1980,1983)$ iniciando de este modo la arqueología inferopaleolítica en Asturias en época moderna y constituyendo estas excavaciones a su vez un hito en la Prehistoria asturiana.

Bañugues se encuentra en las coordenadas $43^{\circ} 38^{\prime} 06^{\prime \prime} \mathrm{N}$ y $5^{\circ} 48^{\prime} 29^{\prime \prime}$ W y su depósito geológico fue descrito por $\mathrm{N}$. Llopis Lladó como un depósito con rellenos aluviales y coluvio-aluviales, en una gran vaguada excavada sobre materiales devónicos, calizas de Nieva y pizarras (Llopis Lladó, 1962).

Estos sedimentos, a su vez, forman una terraza de $+2,5$ / 3 metros en la cual se hallan los materiales paleolíticos, terraza que ha sido progresivamente erosionada por el mar dejando a la luz su contenido arqueológico. Esta circunstancia ha posibilitado que el depósito paleolítico se haya ido perdiendo con el paso de los años, hecho provocado por los agentes mecánicos naturales que interactúan en el entorno del mismo.

Las campañas arqueológicas se iniciaron en el verano de 1977 y se prolongaron durante 4 años. Según lo indicado por Rodríguez Asensio (1980) los horizontes fértiles se dividen en dos, el nivel V y el VII, constituido por dos brechas muy similares entre sí, formadas por cantos de pizarra y cuarcita con matriz de limos arenosos.

A su vez los datos aportados por la sedimentología, inducen a pensar en la formación del depósito arqueológico durante un momento frío, lo que en palabras del 


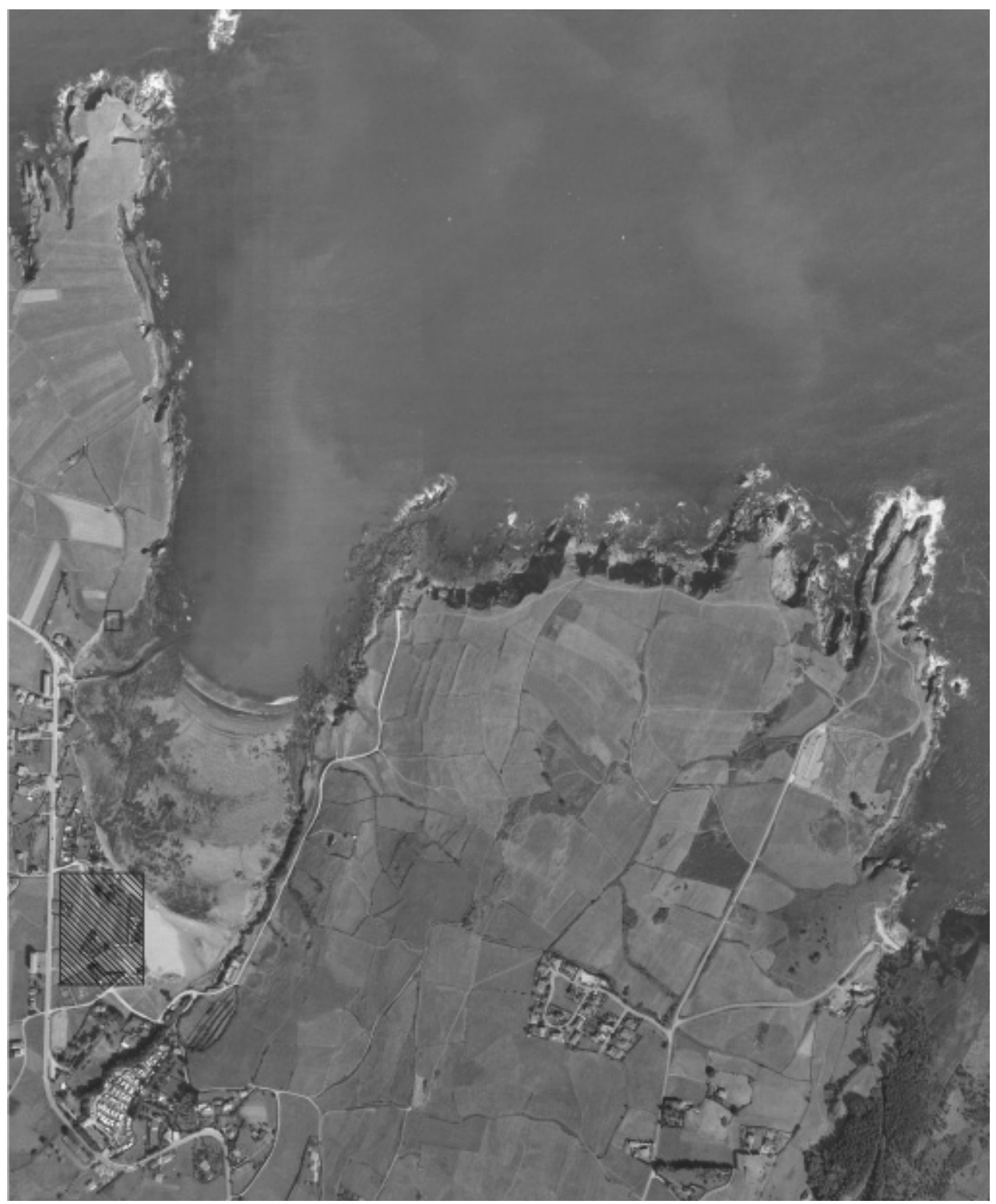

Fig. 2. Situación de Bañugues —Ortofotos del Principado de Asturias 1:5.000—.

profesor Rodríguez Asensio pudo haberse producido hacia el final del interglaciar Riss-Würm o al inicio del Würm I (Rodríguez Asensio, 1990), quedando encuadrado así la formación del depósito en el Ois 5 , aunque etapas con esas características han podido sucederse durante los Ois 6 y 8 e incluso durante algunas pulsaciones frías del Ois 7. 


\section{PROCEDIMIENTO DE ANÁLISIS TECNOLÓGICO}

Se ha procedido a la revisión de una muestra limitada de materiales correspondientes a la excavación de 1977 - se efectuó en el área denominada Sector 1 , en el cual se realizaron 4 catas de $2 \times 2$ metros, de las cuales sólo en una se pudo constatar la presencia de horizontes arqueológicamente fértiles (Rodríguez Asensio, 1980) - con el objetivo de plantear un análisis tecnológico exclusivamente de los productos de talla. Al entender que tradicionalmente en el Paleolítico inferior se han tendido a realizar análisis tipológicos tradicionales, siguiendo las clasificaciones pertinentes que además intentan ser a la vez una herramienta válida para realizar atribuciones cronoestratigráficas, como es el caso de Bordes (1961), Tixier (1956), Echaide (1971), Querol y Santonja (1978) por citar algunas de las más importantes usadas en el Paleolítico inferior peninsular.

Con el análisis tecnológico de los productos de talla pretendemos aprovechar la valiosa información sobre los sistemas de reducción lítica empleados en los conjuntos ínfero paleolíticos, a partir de unos elementos que tradicionalmente han sido desechados, sin contar el plano cuantitativo y espacial, o muy poco tenidos en cuenta a la hora de valorar conjuntamente los datos obtenidos del análisis industrial, centrado sobre manera en los útiles, elementos retocados y en los núcleos. Para el análisis de los métodos de talla y de reducción lítica, tradicionalmente se han utilizado los núcleos, pero estos son elementos que en muchas ocasiones ofrecen una información sesgada e incompleta de los modos de talla existentes, ya que se trata en su mayoría de núcleos que nos han llegado en sus últimas fases de explotación y por lo tanto no se puede extraer más información de la que ofrecen las últimas secuencias de extracción. En cambio los restos de talla más frecuentemente hallados, lo que conocemos por lascas, son un clarísimo testimonio de los procesos de talla y de las distintas estrategias de reducción lítica empleadas, analizando los anversos, la relación angular de los talones, la proporción cortical presente, o los tipos de talón presentes.

Desde este planteamiento creemos fundamental para el entendimiento de las industrias líticas en el Paleolítico antiguo, no sólo su dimensión tecnológica en sentido estricto a nivel cultural, sino también en el aspecto económico y social, los cuales sin lugar a dudas forman parte de los procesos de elaboración de herramientas líticas dentro de los grupos humanos del Paleolítico inferior. En este apartado es importante mencionar los planteamientos de partida del Sistema Lógico Analítico (Mora, Martínez y Terradas, 1991), que recogen en gran parte la propuesta de análisis industrial de Clark (1977) y en la misma línea de trabajos anteriores como los de Isaac (1984). También es importante la introducción en el debate de las industrias del concepto de cadena operativa (Pellegrin et al., 1988), el cual sin duda significó un antes y un después en la concepción de los análisis de los conjuntos industriales paleolíticos, abriendo de una manera definitiva el análisis tecnotipológico a las dimensiones social, económica y cultural de los grupos humanos. 
Para conceptos determinados como los correspondientes a la talla levallois, hemos recurrido a las primeras definiciones (Bordes, 1980) y a los posteriores, más completos y exhaustivos trabajos de Böeda $(1988,1990$ y 1993).

\section{MÉTODOS DE REDUCCIÓN LÍTICA EN BAÑUGUES: CAMPAÑA 1977}

De todo el material estudiado una buena parte no ha podido ser diagnóstico, ya que se trata de restos de talla o lascas simples sin elementos claros para efectuar un análisis tecnológico. Según el estudio de la industria lítica los materiales fueron asignados a un Achelense final / Musteriense de tradición achelense (Rodríguez Asensio, 1983), nosotros hemos desechado el estudio de los elementos retocados y de configuración para complementar esa adscripción cronocultural, centrándonos en los productos de talla con el objetivo de identificar las cadenas operativas existentes.

La mayoría de los talones son lisos, no corticales, y los anversos poseen más de tres extracciones. No hay casi anversos corticales, por lo que a priori podemos entender que se trata de elementos desbastados en otro lugar y aportados al yacimiento, bien como materia prima, bien como productos ya finalizados, aunque el escaso número de piezas retocadas y un número considerable de piezas finales (núcleos agotados, elementos de deshecho, debrises) nos hace pensar en la realización prácticas de debitado en el lugar.

Independientemente de la presencia de distintas materias primas y del diferente grado de calidad de la misma, hay una preponderancia de lascas cortas y anchas, con sus dos caras casi paralelas, en las que el ángulo de percusión varía, lo cual hace que su talón sea normalmente mayor de $90^{\circ}$, por lo tanto debemos adscribir estas lascas a temas operativos de origen bifacial y que dadas las características de conjunto de las lascas se dividan entre los métodos discoide y levallois.

El aprovechamiento de los núcleos parece no tener un patrón uniforme, más bien la talla centrípeta en este caso se usa con la recurrencia consabida para ir aprovechando cada superficie de lascado como plano de percusión para el siguiente levantamiento, núcleos discoides, existiendo un aprovechamiento bastante alto.

También se aprecia la existencia de percutor blando o elástico (ya observado por Rodríguez Asensio, 1980), y la existencia de varios accidentes de talla, como los sobrepasados y las fracturas por flexión en la zona distal.

Existe en este conjunto una selección de la materia prima, la cuarcita, de mayor calidad, es sobre la que se realizan los productos levallois, así como las únicas piezas de sílex.

El estado de conservación de las piezas es variado, aparecen distintos tipos de materia prima (sílex, cuarcita armoricana y de los cabos, cuarzo, areniscas, etc.) y en distintos estados de alteración (puede que por la exposición al medio) lo cual puede, 
porque no, ser un indicador del espectro temporal abarcado (no una única ocupación, puede que un asentamiento temporal, pero prolongado en el tiempo, recurrente).

El hecho de que haya núcleos sobre lascas de pequeñas dimensiones, indica que hay un aprovechamiento intenso de los recursos líticos, lo que nos está indicando una distancia considerable a las fuentes de materias primas (Bernard-Guelle y Porraz, 2001). Se trata de núcleos multidireccionales de pequeñas dimensiones, lo cual implica un aprovechamiento elevado de los recursos líticos.

Los productos de mayor tamaño parecen tener una talla organizada, secuencias de talla recurrentes conducentes al aprovechamiento más efectivo de la materia prima, luego aparecen elementos muy agotados, aprovechados de forma aleatoria, multidireccional, utilizando las extracciones como plataforma de percusión, lo cual nos está indicando el aprovechamiento extremo de los recursos líticos, adaptando el tipo de talla a las características de la materia prima.

Por lo que se refiere al grado de corticalidad del conjunto, podemos indicar que hay varias lascas con corticales distales, lo cual nos habla de la existencia de superficies corticales en los nódulos, pero no totales, que pueden estar previamente desvastados, ya que el número de talones corticales es muy bajo y de corticales proximales casi nulo, hay algún cortical total, pero también se ven pocos.

En definitiva en el conjunto no se observa ninguna lasca que provenga de la talla de bifaces, o elementos similares, el número de útiles es muy pequeño y parece tratarse de un complejo de aprovechamiento de lascas dónde el número y tamaño de los núcleos es también pequeño, síntoma de una explotación intensa en proporción al gran número de lascas, por lo que podemos deducir que o bien faltan núcleos, o la talla se efectuaba en otro lugar, lo cual no es probable dado el elevado número de elementos de deshecho.

Si nos atenemos a la primera conclusión deberemos tener en cuenta la posibilidad de encontrarnos ante el último episodio de una cadena operativa más compleja en la cual el aprovisionamiento y el desbastado primario de los núcleos se realizase en otro lugar, trasladando posteriormente estos elementos a otro lugar en el que se procediese a la extracción de lascas con fines utilitarios y a la fabricación de utillaje retocado.

Existe un aprovechamiento diferencial en función de la materia prima, la menos aprovechada, es decir la que aparece en forma más tosca y peor trabajada (mayor tamaño, menos aprovechada), es una arenisca con grandes granos de cuarzo, y demás nódulos de grano grueso y difícil talla, en cambio existe un aprovechamiento extremo de otras materias primas. El sílex se aprovecha con una talla muy limitada, seguramente aprovechando los soportes de manera natural, debido a su fácil talla y a la calidad de la misma, se aplica solamente retoque.

El modo de reducción empleado de forma más recurrente que se ha observado en el conjunto es el centrípeto, tanto en la talla discoide, mayoritaria, como en la ta- 

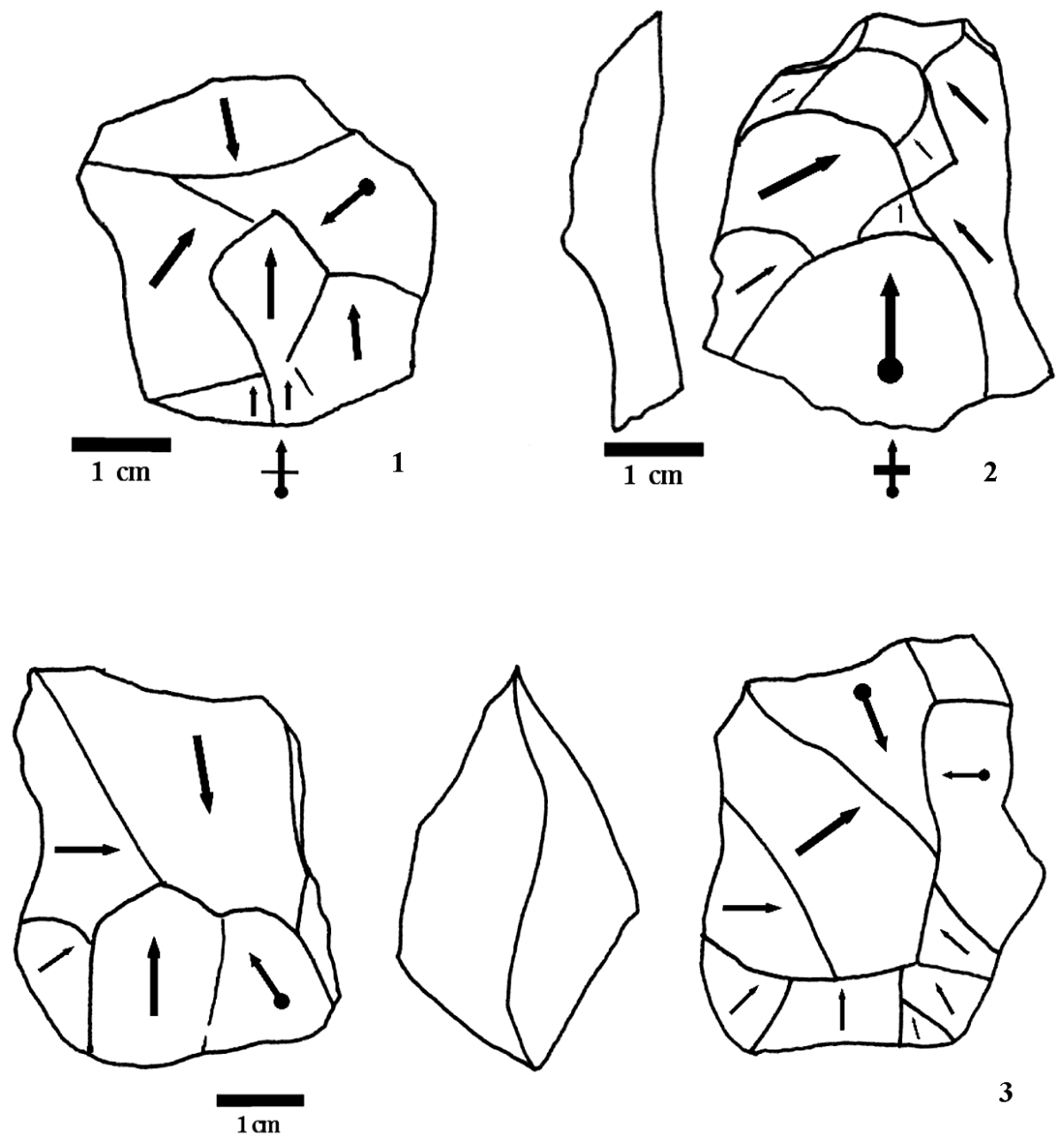

3

Fig. 3. 1 y 2 lascas, 3 núcleo discoide.

Ila levallois. Como hemos visto anteriormente existe una presencia notable de elementos levallois, por el contrario los núcleos de este tipo son inexistentes. Tal vez por agotamiento de los núcleos levallois y de la recurrencia, estos se reconvierten en discoides, aprovechando la materia prima más óptima hasta su agotamiento.

Vemos pues como la ausencia de núcleos levallois nos puede llevar a un error de percepción, en cambio con la presencia de lascas extraídas mediante este sistema podemos llegar a la conclusión de que los sistemas de reducción preferentes son los que utilizan la preparación del soporte y están encaminados a obtener un elemento de características predeterminadas y predefinidas (levallois), continuando con el aprovechamiento del núcleo y la extracción de lascas una vez que ya ha dejado de ser posible la preparación y la preferencia en la talla, pro- 

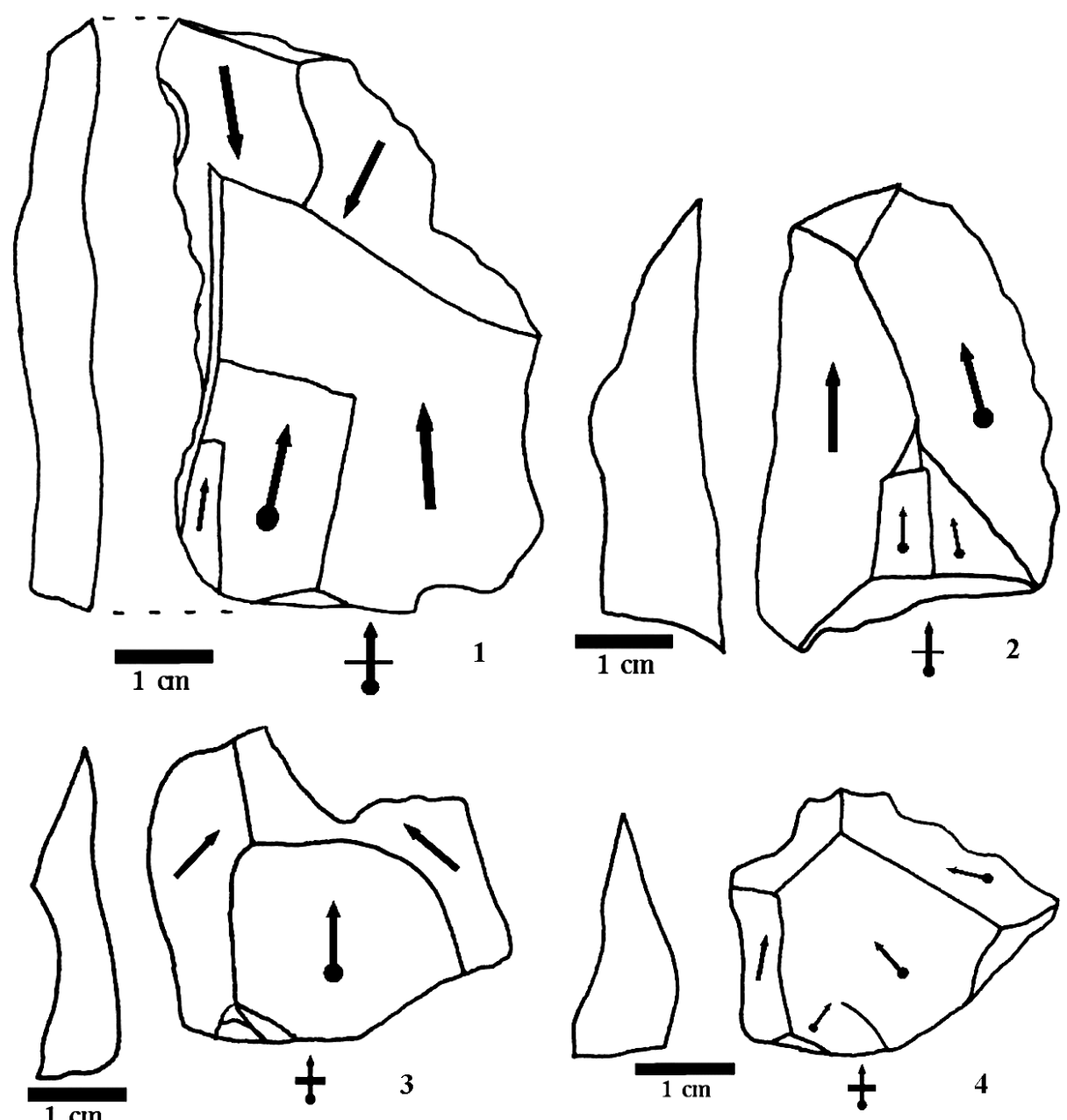

Fig. 4. Lascas Levallois.

siguiendo con el modo de talla más natural que procede en este caso, una talla bifacial no preferencial que da como resultado un núcleo discoide. Así los núcleos levallois pasan a ser núcleos discoides, desapareciendo esta evidencia dentro del grupo de los núcleos.

Destaca la existencia de cadenas operativas que se adaptan al soporte y modifican sus características en función del mismo, así la cadena operativa que comienza siendo levallois puede pasar a ser discoide una vez que el núcleo ya no puede jerarquizarse para la extracción de lascas predeterminadas y una vez imposibilitada la recurrencia de las extracciones. Estas cadenas operativas son complejas, comenzando con la elección de soportes adecuados y probablemente con su desbastado y preparación en el lugar de captación, siendo a continuación 
transportados al yacimiento con un ahorro de esfuerzo y con la posibilidad de llevar consigo más nódulos aptos para la talla inmediata. En el entorno de Bañugues hay una notable cantidad de materia prima, pero las cuarcitas y el sílex en los que se ha procedido a realizar la extracción de lascas predeterminadas no se encuentran en el mismo lugar, no ocurre así en cambio con el macroutillaje.

\section{CONCLUSIONES}

En Bañugues tenemos modo 1, modo 2 y modo 3, con la presencia de técnicas de talla que no son propias del Achelense pero que comienzan a predominar en Europa en torno al 300.000 BP (Moncel et al, 2005) -en esas fechas se está dando el Achelense en Europa- hace más aconsejable una denominación cultural para estos materiales dentro de un Paleolítico medio en sentido amplio, no restringido al Musteriense, como erróneamente se tiende a generalizar. Esta evolución tecnológica que asociamos al Paleolítico medio tiene lejanos antecedentes en África, dónde en el yacimiento olduvayense de Peninj se documentan procesos, que sin ser asimilables a la técnica levallois, comparten los mismos procesos cognitivos, el mismo conocimiento técnico y la misma destreza en las estrategias de talla (de la Torre et al., 2003).

Aunque debemos precisar que si bien podemos ubicar el origen de la técnica levallois en Europa en torno al $300.000 \mathrm{BP}$, no podemos precisar su presencia homogénea en todo el continente en esas fechas.

El motivo de tal diferenciación cultural entre Achelense y Paleolítico medio, dentro del P. antiguo, se debe a que a pesar de ser esta última una denominación apta y válida para los tecnocomplejos líticos que derivan en el Musteriense típico, dando muestra de la evolución sin rupturas que se vive en todo el Pleistoceno medio europeo, se hace necesario una aclaración para no acabar cayendo en el error de meter en un mismo saco cosas que no son comunes, de hacer las matizaciones oportunas, por ello consideramos más correcto reservar la denominación de Achelense a los conjuntos que presentan una talla bifacial incontestable y cuyos elementos contienen las características del Achelense africano, siendo el paso evolutivo en la realización de las industrias más a tener en cuenta, la aparición de sistemas de talla más complejos como el discoide y sobre todo el levallois. Esto tal vez requiera atrasar las cronologías relativas existentes, basadas en la industria lítica, con las que se asigna a los yacimientos del Paleolítico inferior cantábrico, como últimamente está sucediendo con los conjuntos de la meseta como Ambrona (Falguères et al., 2006b), y denominar Paleolítico medio «sensu estrito» con las matizaciones pertinentes a los tecnocomplejos que a partir del Achelense comienzan a registrar ciertas innovaciones tecnológicas, haciéndose presente el modo 3 de reducción lítica y otros métodos similares que implican una talla lítica predeterminada y con preparación previa de los soportes. 
En este contexto, en el que indudablemente está presente el utillaje típico del Paleolítico inferior y que ya se aleja del Achelense para pasar a formar parte del Paleolítico medio, es donde se debe insertar el tecnocomplejo lítico de Bañugues. El hecho de cambiar su denominación de Achelense a Paleolítico medio no implica rejuvenecer el registro, sino todo lo contrario, ya que al Achelense deben atribuírsele cronologías más elevadas, haciendo presencia el Paleolítico medio ya en los estadios finales del Pleistoceno medio. A falta de dataciones absolutas para el yacimiento de Bañugues, creemos que no sólo mantiene la cronología relativa atribuida en su día, sino que también pudiera corresponder a estadios cronológicos anteriores.

Rodríguez Asensio hace una atribución cronológica de la industria de Bañugues basándose en el análisis de la industria lítica y apoyándose en las consideraciones sedimentológicas de $\mathrm{M}$. Hoyos, que asigna una edad eemiense al depósito, o lo que es lo mismo, lo sitúa en el interglaciar Riss-Würm (Hoyos et al., 1977), considerando que se trata de un Achelense superior final.

A día de hoy el Achelense no podemos considerarlo como una cultura que ha pervivido hasta el inicio del Pleistoceno superior, ya que como hemos visto son numerosos los indicios que nos hablan de constantes cambios ocurridos en el seno de la cultura achelense en Europa y que retrasan progresivamene su cronología (Falguères et al., 2006b; Mariani-Constantini et al., 2001; Villa, 2001) que lleva a su paulatina y constante modificación hasta derivar en el Musteriense.

Si bien consideramos, que a raíz de los últimos hallazgos paleoantropológicos podemos hablar de una Europa con presencia de Preneandertales en torno al 300.000 (Agustí y Lordkipanidze, 2005; Bermúdez de Castro et al., 2004; Díez Martín, 2005; Falguères et al., 2004; García Sánchez, 2004, 2006a y 2006b; Huguet, 2004; Moncel et al., 2005) todo ello nos hace incidir en explicitar la terminología cultural empleada hasta el punto de establecer las diferenciaciones oportunas. No obstante, el Achelense hace su aparición en África en contextos cronológicos en torno a los 1.6 / $1.4 \mathrm{~m}$.a donde se documenta cierta coetaneidad del Olduvayense final con el Achelense inicial (de la Torre, 2004; de la Torre et al., 2003) y en 1.5 inició su expansión fuera de África, como lo demuestran los hallazgos del Levante mediterráneo, donde se documentan niveles del Achelense inferior en el yacimiento de Ubeidiya en torno a los 1.4 m.a. (Ronen, 2006).

En otro aspecto, y por lo que se refiere a nuestra área de estudio, la cueva cantábrica de Lezetxiki (Arrasate, Guipúzcoa) ofrece dataciones para los niveles Musterienses en torno a $100.000 \mathrm{BP}$, siendo diagnóstica la datación U/Th de Lezetxiki II con $117+15$ / - 13 ka BP (IPH-Lz16) (Falguères et al., 2006a), viendo que en cronologías cercanas al interglaciar Riss-Würm tal vez ya no sea oportuno seguir hablando de Achelense. Si somos conscientes de las diferencias y de la evolución que observamos en las industrias, no podemos seguir conservando impertérritamente el mismo patrón de división cronocultural establecido a partir de los clásicos postulados tipológicos, creados con una clara vocación evolutiva, como es el caso 
de la columna vertebral del Paleolítico antiguo definida por F. Bordes (1961), ya que el mismo se hace cada vez más insostenible a tenor de la realidad ante la que nos encontramos. La secuencia cronocultural se va retrasando paulatinamente haciéndola corresponder con una cronoestratigrafía más acorde con el origen africano y su posterior dispersión por el continente euroasiático, como queda patente con las continuas revisiones sobre el poblamiento europeo y el debate de la short chronology que incide en un poblamiento definitivo y efectivo del continente por parte de grupos con cultura achelense en torno al 500.000 BP (Denell, 2003; García Sánchez, 2006a, 2006b; Roebroeks y Van Kolfschoten, 1995; Santonja, 1996).

En definitiva optamos por incluir el conjunto de Bañugues dentro de un contexto Paleolítico medio, conservando sus atribuciones cronoestratigráficas que le asignan una edad eemiense, por no ser incoherente con el contexto general del Paleolítico antiguo, e incluso valorando la posibilidad de que su cronología pueda ser anterior. La presencia de bifaces en el yacimiento es muy escasa y aunque se tienda a recurrir al Achelense para la atribución cultural en base a la presencia de estos elementos en los conjuntos líticos, no dejan de ser un elemento más del registro, el cual hay que valorar en todos sus aspectos. Por otra parte las características «abbevillenses» de la indstria bifacial son discordantes con respecto al conjunto lítico complementario, lo cual pudiera llevarnos, a falta de estudios más exhaustivos, a pensar que más que de útiles bifaciales podría tratarse de auténticos núcleos de lascas bifaciales, reabriendo un debate largamente tratado a cerca de las carácterísticas técnicas de los bifaces. La atribución cultural realizada por Rodríguez Asensio (1983), que lo sitúa en un horizonte comprendido entre el Achelense superior final y el Musteriense de tradición achelense constituye una definición muy correcta y ajustada a tenor de las evidencias existentes, lo que sucede es que a día de hoy se hace necesario realizar los oportunos reajustes como hemos mencionado y explicado a lo largo del texto precedente, dado el gran número de hallazgos arqueológicos y paleoantropológicos acaecidos desde entonces, así como la constante revisión a la que se ven sometidos los estudios sobre Paleolítico inferior.

\section{BIBLIOGRAFÍA}

Agustí, J. y LoRdkIPANIDZE, D. (2005): De Turkana al Cáucaso, Ed. National Geographic.

BernaRD-Guelle, S. y Porraz, G. (2001): «Amincissement et débitage sur éclat: définitions, interpretations et discussions à partir d'industries lithiques du Paléolithique moyen des Préalpes du nord françaises", PALEO, 13, pp. 53-72.

Bermúdez de Castro, J. M., Márquez, B., Mateos, A., Martinón-Torres, M. y Sarmiento, S. (2004): Hijos de un tiempo perdido. La búsqueda de nuestros orígenes, Ed. Ares y Mares.

BoËDA, E. (1988): «Le concept Levallois et évaluation de son champ d'application», L'homme de Neandertal, 4, LA TECHNIQUE, Liege, pp. 13-26.

- (1993): «Le débitage discoïde et le débitage Levallois récurrent centripète», Bulletin de la Société Préhistorique Française, 90 (6), pp. 392-404.

- (1994): Le concept Levallois: variabilité des méthodes, CNRS, Paris.

-, Geneste, J-M. y MeIGnen, L. (1990): «Identification de chaines operatoires lithiques du paleolithique ancien et moyen», Paléo, 2, pp. 43-80. 
Bordes, F. (1961): Typologie du Paléolitique ancien et moyen, Delmas, 2 vol., Bourdeaux.

- (1980): «Le débitage Levallois et ses variants», Bulletin de la Société préhistorique française, 77 (2), pp. 45-99.

CLARK, J. G. D. (1977): World Prehistory: A new perspective, Cambridge University Press.

DENNELL, R. (2003): «Dispersal and colonisation, long and short chronologies: how continueous is the Early Pleistocene record for hominids outside East Africa?», Journal of Human Evolution, 45, pp. 421. 440.

Díez MARTín, F. (2005): El Largo viaje. Arqueología de las primeras migraciones humanas, Bellaterra.

ECHAIDE, M. ${ }^{a}$ D. (1971): «La industria lítica del yacimiento de Budiño (Pontevedra. España)», Munibe, XXIII (1), pp. 125-154.

Falguères, Ch., Yokoyama, Y., Shen, G., Bischoff, J. L., Ku, T-L. y Lumley, H. de (2004): «New U-series dates at de Caune de l'Arago, France», Journal of Archaeological Science, 31, pp. 941-952.

Falguères, Ch., Yokoyama, Y. y ArRizabalaga, A. (2006a): «La Geocronología del yacimiento pleistocénico de Lezetxiki (Arrasate, País Vasco). Crítica de las dataciones existentes y algunas nuevas aportaciones», Homenaje a Jesús Altuna. Arqueología, Munibe, 57 (2), pp. 93-106.

Falguères, Ch., Bahain, J. J., Pérez, A., Mercier, N., Santonja, M. y Dolo, J. M. (2006b): «The Lower Acheulian site of Ambrona, Soria, (Spain): ages derived from a combined ESR / U-series model», Journal of Archaeological Science, 33, pp. 149-157.

GaRCíA SÁnCHEZ, E. (2004): «El poblamiento más antiguo de la Península Ibérica. Una revisión, una propuesta cronoestratigráfica y algunas reflexiones sobre la colonización de Eurasia», Miscelánea en homenaje a Emiliano Aguirre. Arqueología, Zona Arqueológica, 4, pp. 179-194.

- (2006a): El primer poblamiento de Europa. Su significado en el contexto de la evolución humana y su relación con la dispersión de homínidos por el viejo mundo. Tesis doctoral inédita, Departamento de Prehistoria y Arqueología, UNED, Madrid.

- (2006b): «El poblamiento de Europa en torno al límite Matuyama / Brunhes: su origen y su significado en la evolución humana», Trabajos de Prehistoria, 63 (1).

GonzÁLez FeRnÁNDEZ, J. M. (1968): «El Paleolítico inferior y medio en Asturias. Nuevos hallazgos», Archivum, XVIII, pp. 1-36.

Hoyos, M., AguirRe, E., Pérez González, A., y Zazo, C. (1977): Donées récentes sur le milieu et l'ocupation humaine au Würm. Approche écologique de l'homme fossile. Travaux du groupe Ouest de l'Europe de la Commission internationale de l'INQUA. Palecology of Early Man (1973-1977) sous la direction de H. Laville y Renault-Miskovsky, J. París. Supplément au Bulletin AFEQ, 47, p. 118.

HUGUET, R. (2004): «Las primeras ocupaciones humanas de Europa: una aproximación al estado de la cuestión», Miscelánea en homenaje a Emiliano Aguirre. Arqueología, Zona Arqueológica, 4, pp. 239-251.

ISAAC, G. L. (1984): «The archaeology of human origins: studies of the Lower Pleistocene in East Africa 1971-1981», en Wendorf, F. y Close, A. (eds): Advances in Old World Archaeology, Academic Press, New York, pp. 1-87.

Jordá Cerdá, F. (1975): «Notas sobre el Asturiense en el Paleolítico Hispano», Las Ciencias, XL (42).

LLOPIS LLADÓ, N. (1962): Mapa geológico de Asturias, Hojas 1 y 2, Estudio de la Región del Cabo de Peñas, Inst. Geol. Min. Esp., Oviedo.

Mariani-Constantini, R., Ottini, L., Caramiello, S., Palmirotta, R., Mallegni, F., Rossi, A., Frati, L. y CaPASSO, L. (2001): «Taphonomy of the fosil hominid bones from the Acheulean site of Castel di Guido near Rome, Italy", Journal of Human Evolution, 41, pp. 211-225.

Moncel, M-H., MoIGNE, A-M. y COMBIER, J. (2005): «Pre-Neandertal behaviour during isotopic stage 9 and the beginning of stage 8 . New data concerning fauna and lithics in the different occupation levels of orgnac 3 (Ardèche, South-East France): ocupation types", Journal of Archaeological Science, 32, pp. 1283-1301.

Mora Torcal, R., Martínez Moreno, J. y Terradas Batlle, X. (1991): «Un proyecto de análisis: El sistema lógico analítico (SLA)", Treballs d’Arqueología I. Tecnología y cadenas operativas líticas, UAB, pp.173-199.

Pelegrin, J., KarLin, C. y Bodu, P. (1988) : :Chaînes opératories: un outil pour le préhistorien», Technologie préhistorique, CNRS, París, pp. 55-62.

Pérez PÉREZ, M. (1975): «Los yacimientos prehistóricos de la región del Cabo de Peñas», XIII Congreso Nacional de Arqueología, Zaragoza, pp. 109-118.

QUEROL, M. ${ }^{\text {a A }}$. y SANTONJA, M. (1978): «Sistema de clasificación de cantos trabajados y su aplicación en yacimientos del Paleolítico antiguo de la Península Ibérica», Saguntum, 13, pp. 11-38.

Rodríguez Asensio, J. A. (1978a): «Nota preliminar sobre las excavaciones del yacimiento de Bañugues (Gozón. Asturias)», BIDEA, 93-94, pp. 357-368. 
- (1978b): «The Early Palaeolithic site of Bañugues (Gozón. Asturias. Spain)», Current Anthropology, 19 (3), pp. 615-616.

- (1980): «Bañugues (Gozón. Asturias). Yacimiento del Paleolítico Inferior», Noticiario Arqueológico Hispánico, 9, pp. 11-31.

- (1983): "La presencia humana más antigua en Asturias», Estudios de Arqueología Asturiana, 2.

- (1990): «Los orígenes del poblamiento en Asturias: El Paleolítico inferior y medio», en Historia de Asturias I, Ed. Prensa Asturiana, pp. 33-48.

- (2004): «¿Territorios en el Paleolítico antiguo asturiano?», Miscelánea en homenaje a Emiliano Aguirre. Arqueología, Zona Arqueológica, 4, 2, pp. 432-439.

Roebroeks, W. y Van Kolfschoten (eds) (1995): The earliest Occupation of Europe. Proceedings of the European Science Foundation Workshop at Tautavel (France), 1993, Leiden Univ. Press, Leiden.

Ronen, A. (2006): «The oldest human groups in the Levant», C.R. Palevol, 5.

SANTONJA, M. (1996): «The lower Paleolithic in Spain: sites, raw material and occupation of the land», en Moloney, Raposo y Santonja (eds): Non-Flint Stone Tools and the Paleolithic Occupation of the Iberian Peninsula, BAR International Series, 649, pp. 1-20.

TIXIER, J. (1956): «Le hachereau dans l'Acheuléen nor-africain. Notes typologiques», Congress. Preh. Fr., XV, Poitiers-Angouleme, pp. 914-923.

TORRE SAINZ, I. de la (2004): Estrategias tecnológicas en el Pleistoceno inferior de África Oriental (Olduvai y Peninj, Norte de Tanzania), Universidad Complutense, Madrid, Tesis doctoral microfilmada, 612 pp.

-, Mora, R., Domínguez-Rodrigo, M., Luque, L. y AlCalÁ, L. (2003): «The Oldowan industry of Peninj and its bearing on the reconstruction of the technological skills of Lower Pleistocene hominids", Journal of Human Evolution, 44, pp. 203-224.

VILLA, P. (2001): «Early Italy and the colonization of Western Europe», Quaternary International, 75, pp. 113-130. 